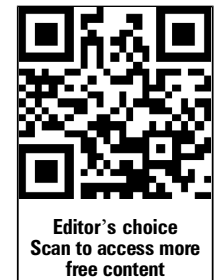

free content

\begin{abstract}
${ }^{1}$ The Ludwig Boltzmann Institute for Retinology and Biomicroscopic Laser Surgery, Vienna, Austria

${ }^{2}$ Department of Ophthalmology, Rudolph Foundation Clinic, Vienna, Austria

${ }^{3}$ Center for Medical Statistics, Informatics, and Intelligent Systems, Section for Medical Statistics, Medical University, Vienna, Austria
\end{abstract}

\section{Correspondence to} Dr Ilse Krebs,

Department of Ophthalmology, Rudolf Foundation Clinic, Juchgasse 25, Vienna A1030, Austria; Ilse.Krebs@wienkav.at

Received 7 April 2013 Revised 8 July 2013 Accepted 24 July 2013 Published Online First 21 August 2013

\footnotetext{
To cite: Krebs I, Glittenberg C,

Ansari-Shahrezaei S, et al.

Br J Ophthalmol

2013;97:1443-1446.
}

\title{
Non-responders to treatment with antagonists of vascular endothelial growth factor in age-related macular degeneration
}

\author{
Ilse Krebs, ${ }^{1,2}$ Carl Glittenberg, ${ }^{1,2}$ Siamak Ansari-Shahrezaei, ${ }^{1,2}$ Stefan Hagen, ${ }^{1,2}$ \\ Irene Steiner, ${ }^{3}$ Susanne Binder ${ }^{1,2}$
}

\begin{abstract}
Purpose Most of the publications on modern therapy of neovascular age-related macular degeneration focus on the effect of the treatment. The purpose of this study is to determine the frequency of non-responders to anti-vascular endothelial growth factor (anti-VEGF) treatment and find possible reasons for their failure to respond.
\end{abstract}

Methods The records of patients treated until the end of 2008 the first time with either bevacizumab or ranibizumab were reviewed. Based on the availability of measurable results and according to prior publications showing the effect of the therapy, loss of three lines of distance acuity, increase of retinal thickness or lesion size were identified as indicators of non-responders. Two of these three signs had to be present.

Results 334 eyes of 283 patients were included; $74.55 \%$ received bevacizumab and $25.45 \%$ received ranibizumab. Overall $14.37 \%$ of the eyes were identified as non-responders (14.06\% in the bevacizumab group and $15.29 \%$ in the ranibizumab group). Baseline distance acuity and vitreo-retinal adhesions were significantly correlated with non-responders. Correlations with age, gender, lesion type, other morphologic features, and the kind of anti-VEGF agent failed to be significant. $10.4 \%$ of the non-responders showed a delayed but good response to anti-VEGF treatment.

Conclusions About 15\% did not sufficiently respond to anti-VEGF treatment. Vitreo-retinal adherences were the only ophthalmologic factor which could be identified to be significantly correlated with insufficient response.

\section{INTRODUCTION}

Intravitreal injections with antibodies of the vascular endothelial growth factor (anti-VEGF) have been established for the treatment of neovascular age-related macular degeneration (AMD) around the world. Today the most frequently applied substances are ranibizumab (Lucentis, Novartis, Basel, Switzerland; approved for the therapy of AMD) and bevacizumab (Avastin, Genentech, San Francisco, USA; used off label for intravitreal injection). Results are available from large multicentre trials focusing on the effect of ranibizumab treatment, ${ }^{12}$ or comparing the efficacy of both substances. ${ }^{3-5}$ However, publications concerning the insufficient response to anti-VEGF treatment are rare and clearly defined criteria to identify non-responders have not yet been published. The aim of this study was to define criteria to identify non-responders, to examine the incidence, and to evaluate possible predictive factors in patients treated with the most frequently applied anti-VEGF agents ranibizumab and bevacizumab.

\section{METHODS}

The records of all patients with active neovascular AMD, who received their first injection with either ranibizumab or bevacizumab between January 2006 and December 2008, were retrospectively reviewed. The data from the baseline examination, and the month 3 and month 12 examinations were evaluated. The results of the month 3 examination were examined to identify non-responders, and the results of the month 12 examination to assess delayed response.

Bevacizumab $1.25 \mathrm{mg}$ was applied in our department until the approval of ranibizumab in the European Union in January 2007. Thereafter, ranibizumab $0.5 \mathrm{mg}$ was commonly used but bevacizumab was also available. The treating physician decided which substance should be used. Patients were also informed about the off label use and received ranibizumab when they insisted on receiving it. The patients were re-treated monthly following a flexible as needed regimen based on optical coherence tomography (OCT) and best corrected visual acuity (BCVA). Presence (increase or persistence) of intra- or subretinal fluid in the macula or loss of more than five letters of BCVA, or signs of activity in biomicroscopy (like new choroidal neovascularisation (CNV) or blood) were the re-treatment criteria. Usually follow-up examinations were performed monthly as long as treatment was required, and thereafter at the discretion of the examiner and at month 12 .

BCVA was tested with ETDRS (Early Treatment Diabetic Retinopathy Study) charts at $2 \mathrm{~m}$ distance before treatment and monthly thereafter. The pupils were dilated and the OCT examinations were performed with the Stratus OCT until 2007 and thereafter with the Cirrus OCT (both instruments Zeiss, Dublin, USA). When Stratus OCT was initially used in a patient it was also used at the follow-up visits even when the Cirrus OCT was available. The retinal thickness programme of Stratus OCT and the cube $512 \times 128$ programme of Cirrus OCT were applied. To assess the activity of a lesion the OCT scans were explored, and increased activity in the OCT scans and automatically recorded central retinal thickness (CRT) were used to identify non-responders. CRT values of Stratus OCT were converted to Cirrus OCT levels to provide comparable data. ${ }^{6}$ The fluorescein angiograms were performed with the Heidelberger 
Retina Angiograph (Heidelberg engineering, Heidelberg, Germany) at baseline and thereafter at the discretion of the examiner (in cases of new blood or new choroidal neovascularisation, and loss of distance acuity).

Evaluation of the morphologic changes in the OCT and fluorescein angiography (FA) examinations, retinal thickness measurement, and assessment of BCVA were performed by different independent examiners. Besides the type of the lesion, special morphologies were assessed possibly influencing the response: haemorrhage or fibroses of more than one disk area and vitreomacular adhesions surrounded by shallow detachment of the posterior vitreous.

To identify eyes not sufficiently responding to treatment, examinations providing measurable data were chosen: BCVA, CRT, and lesion size. A loss of BCVA of at least three lines (in accordance with prior studies ${ }^{1}{ }^{2}$ ), absence of a decrease of CRT, and an increase in lesion size were the criteria. Because of the possible variability of these values, which might not be pathology related, it was decided that two of these parameters had to be present.

\section{Statistical method}

The primary end point of the study was the dichotomous variable non-responder (yes vs no). Univariate generalised mixed models with patient number as the random factor and the primary end point as the dependent variable were calculated to analyse the effect of the independent variables of type of treatment (bevacizumab/ranibizumab), sex, age, type of lesion, special morphology (haemorrhage, fibrosis, and adherences of the posterior vitreous), BCVA, CRT, and lesion size, at baseline respectively. In case of a significant result, the independent variable was included in a multiple generalised mixed model. The independent variable haemorrhage was analysed by a logistic regression model with Firth correction, as the mixed model did not converge due to complete separation.

To analyse the differences between the treatment groups in the change of BCVA after 3 or 12 months, respectively, compared to baseline, we calculated linear mixed models with BCVA at 3 or 12 months, respectively, as the dependent variable, the BCVA at baseline, the type of treatment (bevacizumab/ranibizumab), sex, age and number of injections as the independent variables, and patient number as random effect. A similar analysis was conducted with CRT at 3 or 12 months, respectively, as the dependent variable.

Descriptive statistics are reported as mean \pm SD for continuous variables and as percentages for categorical data.
Statistical analyses were carried out with the statistics programme SAS V.9.2. For all analyses, the significance level was set to 0.05 .

The data collection was conducted in compliance with the tenets of the Declaration of Helsinki.

\section{RESULTS}

Three hundred and thirty-four eyes of 283 patients received the first anti-VEGF injection in the given period and were therefore selected; 100\% completed month 3 and 97\% month 12 examination. The mean age of patients was $76.29 \pm 8.9$ years; $37.8 \%$ were male, $62.2 \%$ female; $74.55 \%$ received primary bevacizumab, and $25.45 \%$ ranibizumab. There were mean $4.8 \pm 2.1$ injections administered per eye up to month 12 , mean $4.7 \pm 2.1$ of bevacizumab and mean $5.3 \pm 2.0$ of ranibizumab, respectively. Overall $14.37 \%$ of the eyes were identified as non-responders, $14.06 \%$ in the bevacizumab group and $15.29 \%$ in the ranibizumab group. Activity of the lesion was identified in $64.7 \%$ of the eyes at month 3 indicating the need for re-treatment. In 100\% of the eyes presenting with increased CRT there was increased activity compared to baseline. Distribution of sex, age, different types of lesion (occult, occult with detachment of the retinal pigment epithelium, classic, and retinal angiomatous proliferation (RAP)), and special morphology (haemorrhage, fibrosis, adherences of the posterior vitreous) in the study eye are presented in table 1.

Univariate analyses revealed a significant effect of adherences of the posterior vitreous $(p=0.0075)$ and baseline BCVA (0.0065) (table 2). The risk of being a non-responder was lower for patients without adherences of the posterior vitreous and worse BCVA. Including these two variables in a multivariate model, the vitreous adherences (OR 0.405 , 95\% CI 0.190 to $0.862, \mathrm{p}=0.020)$ and baseline BCVA (OR 1.014, 95\% CI 1.003 to $1.025, \mathrm{p}=0.013$ ) remained significant.

For the dependent variable BCVA at 3 or 12 months, respectively, a significant effect of BCVA at baseline ( 3 months: estimate $0.84,95 \%$ CI 0.77 to $0.90, \mathrm{p}<0.0001 ; 12$ months: $0.83,95 \%$ CI 0.75 to $0.91, \mathrm{p}<0.0001)$, but no significant effect of treatment ( 3 months: $\mathrm{p}=0.75 ; 12$ months: $\mathrm{p}=0.15)$, age ( 3 months: $\mathrm{p}=0.30 ; 12$ months: $\mathrm{p}=0.57)$, sex (3 months: $\mathrm{p}=0.69$; 12 months: $p=0.96$ ) or number of injections (3 months: $\mathrm{p}=0.75 ; 12$ months: $\mathrm{p}=0.26$ ) was observed.

The linear mixed model with CRT at 3 or 12 months, respectively, as dependent variable revealed a significant effect of CRT at baseline ( 3 months: estimate 0.58 , 95\% CI 0.50 to 0.66 ,

Table 1 Distribution of characteristics of the study eyes

\begin{tabular}{lll}
\hline & Responder $(\mathbf{n}=\mathbf{2 8 6})$ & Non-responder $(\mathbf{n}=\mathbf{4 8})$ \\
\hline Age & $76.5 \pm 8.9$ & $74.7 \pm 8.7$ \\
Sex male, $\mathrm{n}(\%)$ & $109(38.1 \%)$ & $15(31.3 \%)$ \\
BCVA baseline & $48 \pm 31$ & $62 \pm 30$ \\
CRT baseline & $342.3 \pm 100.7$ & $330.4 \pm 88.7$ \\
Occult lesion/PED/classic/RAP, $\mathrm{n}(\%)$ & $103(36.14 \%) / 117(41.05 \%) / 22(7.72 \%) / 43(15.09 \%)$ & $14(29.17 \%) / 17(35.42 \%) / 5(10.42 \%) / 12(25 \%)$ \\
Vitreous adherences yes, $\mathrm{n}(\%)$ & $37(12.94 \%)$ & $14(29.17 \%)$ \\
Haemorrhage yes, $\mathrm{n}(\%)$ & $7(2.45 \%)$ & $0(0 \%)$ \\
Fibrosis yes, $\mathrm{n}(\%)$ & $48(16.78 \%)$ & $11(22.92 \%)$ \\
Bevacizumab/ranibizumab, $\mathrm{n}(\%)$ & $214(74.83 \%) / 72(25.17 \%)$ & $35(72.92 \%) / 13(27.08 \%)$ \\
Number of injections & $4.8 \pm 2.1$ & $5.3 \pm 2.2$ \\
Lesion size & $3.5 \pm 1.7(\mathrm{n}=283)$ & $3.6 \pm 1.4(\mathrm{n}=48)$ \\
\hline
\end{tabular}

BCVA, best corrected visual acuity; CRT, central retinal thickness; PED, pigment epithelial detachment; RAP, retinal angiomatous proliferation. 
Table 2 Results of the univariate generalised mixed models with non-responder (yes/no) as dependent variable

\begin{tabular}{|c|c|c|}
\hline Variable & OR $(95 \% \mathrm{Cl})$ & p Value \\
\hline Treatment (bevacizumab vs ranibizumab) & $0.906(0.445$ to 1.845$)$ & 0.78 \\
\hline Vitreous adherences (no vs yes) & $0.361(0.173$ to 0.752$)$ & 0.0075 \\
\hline Fibrosis (no vs yes) & $0.680(0.317$ to 1.459$)$ & 0.32 \\
\hline Haemorrhage* (no vs yes) $^{*}$ & $2.599(0.120$ to 56.176$)$ & 0.54 \\
\hline Sex (male vs female) & $0.740(0.377$ to 1.451$)$ & 0.37 \\
\hline Side (right vs left) & $1.164(0.620$ to 2.187$)$ & 0.63 \\
\hline Age & $0.978(0.945$ to 1.012$)$ & 0.19 \\
\hline Lesion (occult vs RAP) & $0.485(0.201$ to 1.174$)$ & 0.11 \\
\hline Lesion (PED vs RAP) & $0.520(0.222$ to 1.218$)$ & 0.13 \\
\hline Lesion (classic vs RAP) & $0.818(0.244$ to 2.740$)$ & 0.74 \\
\hline BCVA baseline & $1.015(1.005$ to 1.027$)$ & 0.0065 \\
\hline OCT baseline & 0.999 (0.995 to 1.002$)$ & 0.44 \\
\hline Number of injections & $1.140(0.981$ to 1.325$)$ & 0.087 \\
\hline Lesion size & $1.043(0.874$ to 1.246$)$ & 0.63 \\
\hline
\end{tabular}

$\mathrm{p}<0.0001 ; 12$ months: estimate $0.38,95 \%$ CI 0.28 to 0.47 , $\mathrm{p}<0.0001$ ), but no significant effect of treatment (3 months: $\mathrm{p}=0.16 ; 12$ months: $\mathrm{p}=0.97)$, age $(3$ months, $\mathrm{p}=0.12$; 12 months: $p=0.75)$, sex ( 3 months: $p=0.15 ; 12$ months: $\mathrm{p}=0.53$ ) or number of injections ( 3 months: $\mathrm{p}=0.19 ; 12$ months: $\mathrm{p}=0.99)$.

Regarding the 51 patients who were treated in both eyes, 65\% were responders in both eyes, $4 \%$ non-responders in both eyes, and in $31 \%$ the patients showed a different response in both eyes.

Seventy-seven per cent of the non-responders were identified by a loss of more than 15 letters and an increase of retinal thickness and/or of lesion size. Twenty-three per cent presented an increase of retinal thickness and lesion size and a decrease of distance acuity of less than 15 letters. A delayed but good response reaching at least the baseline distance acuity values showed $7.7 \%$ of the eyes identified as non-responders at month 6 and $10.8 \%$ at month 12 . One patient showed an increase of distance acuity after the detachment of the posterior vitreous. In $16.6 \%$ of patients the anti-VEGF treatment was discontinued after they were identified as non-responders, according to their wishes; the remaining eyes received further intravitreal injections. In $29.2 \%$ of the non-responders bevacizumab was replaced by ranibizumab, and only one eye showed an increase of distance acuity thereafter; $2.1 \%$ received additional reduced fluence photodynamic therapy (PDT), and 2.1\% received a combined intravitreal injection of $4 \mathrm{mg}$ triamcinolone acetonide and bevacizumab. After the PDT the retinal thickness decreased but the distance acuity did not improve, and the eye receiving additional triamcinolone acetonide remained unchanged; $14.6 \%$ showed a slight recovery of distance acuity of no more than five letters, and $7.2 \%$ of the responders exhibited a vision loss of more than 15 letters up to month 12 .

\section{DISCUSSION}

The definition of a sufficient response to treatment differs according to the person doing the evaluating. The patients measure the success of a treatment by their ability to perform their daily tasks such as reading, driving a car, or recognising faces. In contrast the treating physicians use quite different criteria. We have chosen measurable data of examinations most frequently performed in the follow-up of anti-VEGF treatment. The loss of less than three lines of BCVA tested with ETDRS charts was one of the major outcome measures in prior large studies to verify stabilisation. ${ }^{1}{ }^{2}$ Absence of this increase should be a valuable indicator of an insufficient response. Furthermore, this difference is not due to the variability of testing $(95 \%$ CI of repeatability was 17 letters). ${ }^{7}$ Recently, the decrease of retinal thickness measured with OCT has gained importance for verifying the effect of anti-VEGF treatment. ${ }^{3-5} 8$ An increase of lesion size measured during FA is still an important and frequently available parameter to indicate disease progression. Because of the variability of all these data we decided that two of these three parameters had to be present in order to identify a nonresponder. Because the maximum effect of treatment was expected at month 3 , we decided to use the results of the month 3 examination to evaluate the response.

Overall $14.37 \%$ of the eyes were identified as non-responders at month 3, with comparable values for bevacizumab (14.06\%) and ranibizumab (15.29\%). According to the treatment protocol all non-responders had received three anti-VEGF injections, exhibiting vision loss and persistent or increased activity. The incidence of non-responders exceeded the number of eyes losing more than three lines in the MARINA and ANCHOR study. ${ }^{2}$ This had to be expected when including cases that were not selected. Although it seemed to be reasonable to use a combination of functional and morphologic parameters to clearly identify an insufficient response, BCVA was shown to be the most important parameter. Seventy-seven per cent of the non-responders presented a loss of more than three lines, and $83 \%$ an increase of CRT. Twenty-three per cent were identified by OCT and fluorescein angiography only, but they also had a loss of distance acuity of less than three lines.

It is not fully understood why some lesions do not show an effect of anti-VEGF treatment. Although VEGF was identified as playing a key role in the development of neovascular AMD, ${ }^{9}$ other cytokines might play a more prominent role in lesions, revealing less favourable results. ${ }^{10}$ Additionally, the complement system and inflammatory processes contribute to the development of neovascular AMD. ${ }^{11}$ Recently, genetic factors were identified as playing a role in the response to ranibizumab treatment. ${ }^{12}$ However, in bilateral neovascular AMD only 4\% were non-responders in both eyes and $31 \%$ showed a different response in both eyes. Therefore, a local condition different in both eyes has to be suspected to be responsible for the reduced effect of anti-VEGF. In accordance with prior publications in the current study, better baseline distance acuity values were also correlated with the number of nonresponders. ${ }^{13}$ This was expected, because eyes showing good distance acuity have a better chance to lose more than three lines than those with already devastated function. In contrast with the findings of Cohan et al, age and gender did not influence the response to anti-VEGF. ${ }^{14}$ Concerning the lesion type, the percentage of nonresponders was higher in classic lesions and those with RAP. However, the differences failed to be significant. The anti-VEGF agent did not reveal an influence either on the percentage of nonresponders or the distance acuity or retinal thickness outcome at months 3 and 12 .

The only morphologic parameter that significantly correlated with the number of non-responders was the presence of vitreomacular adhesion surrounded by a shallow detachment of the posterior vitreous. As was reported in prior studies, vitreomacular adhesion is associated with neovascular AMD. ${ }^{15}{ }^{16}$ In studies including patients who had neovascular AMD in one eye and dry AMD in the fellow eye to exclude genetic and environment factors, it has been shown that in neovascular AMD these 
adhesions are significantly more frequent. ${ }^{17}$ Ultrasound findings were not available in the current study; therefore completely detached or attached posterior vitreous could not be identified. The favourable effect of vitrectomy or spontaneous posterior vitreous detachment on the activity of the lesion provided further evidence of the impact of vitreo-macular adhesions on the effect of treatment. ${ }^{18} 1916$

In most of the non-responders at month 3 the anti-VEGF therapy was continued. The incidence of delayed but good response up to month 12 to anti-VEGF treatment was $10.4 \%$. This low incidence of delayed response verifies the evaluation of non-responders at month 3 . A change of the anti-VEGF agent from bevacizumab to ranibizumab did not usually improve the results. In contrast to our non-responder cases, in eyes exhibiting tachyphylaxis a change of the anti-VEGF agent was successful in $84 \% .^{20} 21$ Additional PDT immediately reduced intraretinal fluid, but distance acuity was not improved. The combination of anti-VEGF and triamcinolone acetonide was not successful in our study. $^{22} 23$ However, only single cases received combination therapy. Furthermore, the effect of vitrectomy or pharmacologic vitreolysis, especially in non-responders with vitreo-macular adhesions, has not yet been sufficiently examined. ${ }^{24} 25$

The most important limiting factor of the study is its retrospective nature, although a large number of eyes/patients were included. Different examiners decided which substance was used and when re-treatment was necessary. However, this fact did not influence the incidence of non-responders, because due to the re-treatment criteria all of the non-responders had received the maximum number of injections up to month 3. However, these facts and extension of the interval between the scheduled visits after the inactivity was determined could have influenced the effect of the therapy. Therefore we did not present the treatment outcome data for either ranibizumab or bevacizumab or a comparison between both substances.

In summary, about $14 \%$ of the eyes receiving ranibizumab or bevacizumab intravitreal injections were non-responders. Loss of distance acuity and increase of retinal thickness and lesion size at month 3 are appropriate to identify non-responders. Besides baseline distance acuity, the presence of vitreo-retinal adhesion was the only parameter significantly correlated with nonresponders. Alternative therapies have to be developed for the treatment of cases who do not respond sufficiently.

Contributors IK: substantial contribution to conception and design, acquisition of data, analysis and interpretation of data, drafting the article, final approval. CG: substantial contribution to acquisition of data, analysis of data, revising the article, final approval. SH: substantial contribution to acquisition of data, analysis of data, revising the article, final approval. SA-S: substantial contribution to conception and design and acquisition of data, revising the article, final approval. IS: substantial contribution to conception and design, statistical analysis of data, final approval. SB: substantial contribution to conception and design, revising the article, final approval, general supervision of the research group.

Funding Supported by an unrestricted research grant from the L. Boltzmann Institute to SB.

\section{Competing interests None.}

Provenance and peer review Not commissioned; externally peer reviewed.

\section{REFERENCES}

1 Rosenfeld PJ, Brown DM, Heier JS, et al.; MARINA Study Group. Ranibizumab for neovascular age-related macular degeneration. N Engl J Med 2006;355:1419-31.
2 Kaiser PK, Brown DM, Zhang K, et al. Ranibizumab for predominantly classic neovascular age-related macular degeneration: subgroup analysis of first-year ANCHOR results. Am J Ophthalmol 2007:144:850-7.

3 Martin DF, Maguire MG, Ying GS, et al.; CATT Research Group. Ranibizumab and bevacizumab for neovascular age-related macular degeneration. N Engl J Med 2011;364:1897-908.

4 Chakravarthy U, Harding SP, Rogers CA, et al.; AN Study Investigators. Ranibizumab versus bevacizumab to treat neovascular age-related macular degeneration: one-year findings from the IVAN randomized trial. Ophthalmology 2012:119:1399-411.

5 Krebs I, Schmetterer L, Boltz A, et al.; MANTA Research Group. A randomised double-masked trial comparing the visual outcome after treatment with ranibizumab or bevacizumab in patients with neovascular age-related macular degeneration. $\mathrm{Br} \mathrm{J}$ Ophthalmol 2013;97:266-71.

6 Krebs I, Hagen S, Smretschnig E, et al. Conversion of Stratus optical coherence tomography $(\mathrm{OCT})$ retinal thickness to Cirrus OCT values in age-related macular degeneration. Br J Ophthalmol 2011;95:1552-4.

7 Patel PJ, Chen FK, Rubin GS, et al. Intersession repeatability of visual acuity scores in age-related macular degeneration. Invest Ophthalmol Vis Sci 2008;49:4347-52.

8 Fung $A E$, Lalwani GA, Rosenfeld PJ, et al. An optical coherence tomography-guided, variable dosing regimen with intravitreal ranibizumab (Lucentis) for neovascular age-related macular degeneration. Am J Ophthalmol 2007;143:566-83.

9 Adamis AP, Miller JW, Bernal MT, et al. Increased vascular endothelial growth factor levels in the vitreous of eyes with proliferative diabetic retinopathy. Am J Ophthalmol 1994;118:445-50.

10 de Oliveira Dias JR, Rodrigues EB, Maia M, et al. Cytokines in neovascular age-related macular degeneration: fundamentals of targeted combination therapy. Br J Ophthalmol 2011;95:1631-7.

11 Wang Y, Wang VM, Chan CC. The role of anti-inflammatory agents in age-related macular degeneration (AMD) treatment. Eye 2011;25:127-39.

12 Francis PJ. The influence of genetics on response to treatment with ranibizumab (Lucentis) for age-related macular degeneration: the Lucentis Genotype Study (an American Ophthalmological Society thesis). Trans Am Ophthalmol Soc 2011;109:115-56.

13 Menghini M, Kurz-Levin MM, Amstutz C, et al. Response to ranibizumab therapy in neovascular AMD - an evaluation of good and bad responders. Klin Monbl Augenheilkd 2010;227:244-8.

14 Cohen SY, Oubraham H, Uzzan J, et al. Causes of unsuccessful ranibizumab treatment in exudative age-related macular degeneration in clinical settings. Retina 2012:32:1480-5.

15 Krebs I, Brannath W, Glittenberg C, et al. Posterior vitreomacular adhesion: a potential risk factor for exudative age-related macular degeneration? Am J Ophthalmol 2007;144:741-6.

16 Krebs I, Glittenberg C, Zeiler F, et al. Spectral domain optical coherence tomography for higher precision in the evaluation of vitreoretinal adhesions in exudative age-related macular degeneration. Br J Ophthalmol 2011;95:1415-8.

17 Robison CD, Krebs I, Binder $\mathrm{S}$, et al. Vitreomacular adhesion in active and end-stage age-related macular degeneration. Am J Ophthalmol 2009;148:79-82.

18 Liu YL, Lin CP, Yang CM. Transient regression of choroidal neovascularization membrane after vitrectomy in age-related macular degeneration with vitreomacular traction syndrome. Acta Ophthalmol 2011;89:e291-3.

19 Schulze S, Neugebauer A, Kroll P. Appearance of age-related macular degeneration in vitrectomized and nonvitrectomized eyes: an intraindividual case study. Acta Ophthalmol 2008;86:470-81.

20 Gasperini JL, Fawzi AA, Khondkaryan A, et al. Bevacizumab and ranibizumab tachyphylaxis in the treatment of choroidal neovascularisation. $\mathrm{Br} J$ Ophthalmol 2012;96:14-20.

21 Binder $S$. Loss of reactivity in intravitreal anti-VEGF therapy: tachyphylaxis or tolerance? Br J Ophthalmol 2012;96:1-2.

22 Ahmadieh $\mathrm{H}$, Taei R, Riazi-Esfahani M, et al. Intravitreal bevacizumab versus combined intravitreal bevacizumab and triamcinolone for neovascular age-related macular degeneration: six-month results of a randomized clinical trial. Retina 2011;31:1819-26.

23 Tao $Y$, Jonas JB. Intravitreal bevacizumab combined with intravitreal triamcinolone for therapy-resistant exudative age-related macular degeneration. J Ocul Pharmacol Ther 2010;26:207-12.

24 Sebag J. Pharmacologic vitreolysis-premise and promise of the first decade. Retina 2009;29:871-4

25 Benz MS, Packo KH, Gonzalez V, et al. A placebo-controlled trial of microplasmin intravitreous injection to facilitate posterior vitreous detachment before vitrectomy. Ophthalmol 2010;117:791-7. 